\title{
Métrologie et interférences en lumière polarisée
}

\author{
A.C. Boccara et P. Gleyzes \\ Laboratoire d'Optique Physique, UPR A0005 du CNRS, ESPCI, I0 rue Vauquelin, \\ 75005 Paris, France
}

\begin{abstract}
Résumé : Le but de cet article est de montrer comment les notions fondamentales décrivant la polarisation de la lumière peuvent être mises à profit pour concevoir des intruments d'une très grande sensibilité. Nous insisterons partuculièrement sur les effets "d'interférences en lumière polarisée" qui peuvent être exploités pour mettre en évidence des différences de marche de l'ordre du millionième de la longueur d'onde ce qui reste difficile en interférométrie traditionnelle.
\end{abstract}

\section{POLARISATION : LUMIÈRE ET MATIÈRE}

Une description rigoureuse des champs électromagnétiques émis par des ensembles d'atomes passe par une description statistique des effets physiques rendant compte du rayonnement [1]. Le cas des lasers est parfois plus simple quoique quelques précautions doivent être prises lorsqu'il s'agit de rendre compte des interactions du champ avec la matière.

Dans cet exposé nous nous limiterons à quelques notions simples qu'il est bon d'avoir à l'esprit lorsque l'on utilise des montages dont les signaux ont pour origine les effets de polarisation. Ces notions étant très "classiques" (au sens de l'enseignement de l'électromagnétisme) il existe des ouvrages en langue française [2] [3] qui couvrent bien la description "classique" (au sens de la physique quantique) des champs et de leur interaction macroscopique avec la matière.

\subsection{Lumière naturelle et lumière polarisée}

\subsubsection{Sources incohérentes (spatialement).}

Dans ces sources il n'existe pas de corrélations spatiales ou temporelles entre le rayonnement émis par des atomes différents. De ce fait, même si l'énergie moyenne reçue en un point de l'espace ( $r$ ) à partir de la source (placée en $O$ ) est parfaitement stable, il y aura de très rapides fluctuations de la direction du champ électrique de l'onde en $\mathbf{r}$. Les temps caractéristiques sont typiquement de l'ordre de grandeur de la durée des trains d'ondes $\left(10^{-8} \mathrm{~s}\right.$ pour une source à basse pression à $10^{-14} \mathrm{~s}$ pour des sources thermiques).

L'observation que nous faisons est généralement "lente" à cette échelle de temps (oeil, caméra, détecteurs...) ; on pourra rendre compte de l'onde transversale émise par la source 
par la superposition de deux champs $\left(\vec{E}=\vec{E}_{1}+\vec{E}_{2}\right)$ orthogonaux dont nous mesurons la valeur moyenne du carré du module (valeur moyenne prise sur le temps de réponse du détecteur).

Il faut souligner :

- Le choix arbitraire de la base : (par exemple $\vec{E}_{1}$ et $\vec{E}_{2}$ peuvent être choisis selon $\vec{i}$ et $\vec{j}(\vec{i} \perp \vec{j})$ ou selon $\alpha \vec{i}+\beta \vec{j}$ et $\beta \vec{i}-\alpha \vec{j} \forall \alpha, \beta$ pour des polarisations linéaires).

- Le choix arbitraire des deux états de polarisation représentant $\overrightarrow{\mathrm{E}}_{1}$ et $\overrightarrow{\mathrm{E}}_{2}$ (linéaire, circulaire, elliptique).

- La mesure, dans le domaine optique, n'est pas celle du champ, mais les détecteurs (quadratiques) fournissent un signal proportionnel à la puissance reçue intégrée sur le temps de réponse du système de mesure.

Nous appellerons polariseur un système capable d'imposer une différence de phase, $\delta$, constante au cours du temps entre les deux états de polarisation qui nous ont servi de base.

Exemples

$$
\begin{aligned}
& \text { Base cartésienne }(\overrightarrow{\mathrm{i}}, \overrightarrow{\mathrm{j}}) \text {. } \\
& \text { Polarisation linéaire : } \delta=0 \text { modulo } \pi \text {. } \\
& \overrightarrow{\mathrm{E}}=\overrightarrow{\mathrm{E}}_{1}+\overrightarrow{\mathrm{E}}_{2} \text { reste parallèle à une direction fixe au cours de la } \\
& \text { propagation de l'onde. } \\
& \text { Polarisation circulaire : } \delta= \pm \pi / 2 \text { modulo } 2 \pi \text { et } \alpha=\beta \text {. } \\
& \text { Polarisation elliptique : } \delta=\text { constante. }
\end{aligned}
$$

Si nous imposons les axes $\mathrm{x}$ et $\mathrm{y}$, un polariseur linéaire $\mathrm{P}$ imposera la mise en phase $\delta$ des deux composantes (de la lumière naturelle). La direction de la polarisation émergente étant fixée par le rapport des amplitudes selon $x$ et $y$. Remarquons que quelle que soit la direction de polarisation recherchée l'énergie recueillie sera proportionnelle à $\left\langle E^{2}\right\rangle$ $=\left\langle(\alpha \cos \theta+\beta \sin \theta)^{2}\right\rangle$ où $\theta$ est l'angle $(\vec{i}, P)$.

Comme $<\alpha \beta \cos \theta \sin \theta>=0$ on trouve $\left\langle E^{2}>=\frac{1}{2}\left(E_{1}^{2}+E_{2}^{2}\right)\right.$ c'est-à-dire la moitié de l'énergie incidente.

\subsubsection{Sources cohérentes (Lasers).}

Très souvent on a tendance à confondre les effets de cohérence et de polarisation dans le cas des lasers. Remarquons d'abord que si de nombreux lasers fournissent une lumière (fortement) polarisée, c'est dû à des effets d'anisotropie du milieu amplificateur (gain du matériau cristallin pour une direction particulière du champ) ou de la structure (fenêtres, ou milieu amplificateur sous l'incidence de Brewster qui favorise une polarisation comme on le verra plus loin).

Lorsque la symétrie est telle que le système est isotrope perpendiculairement à sa direction de propagation, rien ne devrait favoriser des directions particulières du champ électrique. En fait, pour des raisons technologiques (défauts) ou physiques (effet Zeeman), on observe à la sortie de tels lasers ( $\mathrm{HeNe}$ ) deux polarisations orthogonales stables au cours du temps. Ces deux états de polarisation (linéaires, ou circulaires dans le cas du laser "Zeeman") correspondent à des modes différents qui n'ont pas de relations de phase bien définies mais 
qui, compte tenu de la "longueur des trains d'ondes" (cohérence), peuvent "battre" à des fréquences allant de quelques $\mathrm{MHz}$ (Zeeman) au $\mathrm{GHz}$.

\subsection{La polarisation et les milieux matériels}

Nous n'avons, jusqu'à présent, décrit les effets de polarisation que de façon phénoménologique sans indiquer ce qui pouvait créer, transformer ou détruire un état de polarisation.

Ces effets sont obtenus, par l'interaction de la lumière et de la matière, de très nombreuses façons : réfraction, diffusion, absorption, émission... Nous nous limiterons ici a la description "macroscopique" de ces interactions dans deux cas très classiques : les effets d'interfaces (réflexion, transmission) et les milieux anisotropes (propagation).

Rappelons nous simplement que le comportement des ondes électromagnétiques dans un milieu matériel (linéaire et homogène) ressemble à celui qu'elles ont dans le vide (surtout pour les milieux transparents) mais qu'il faut tenir compte de la polarisabilité du milieu qui intervient par sa constante diélectrique $\left(\varepsilon=\varepsilon_{0} \varepsilon_{r}\right)$ ou par son indice de réfraction $\varepsilon_{r}=n^{2} \varepsilon$ $\varepsilon_{\mathrm{r}}$ et $\mathbf{n}$ sont des grandeurs réelles positives dans le cas des milieux transparents (c'est à dire lorsque la fréquence de l'onde est plus basse que celles des résonances électroniques) et négatives ou complexes dans le cas contraire.

\section{I.2.I Réflexion et transmission à l'interface entre deux milieux isotropes.}

Les relations entre les champs incidents et les champs réfléchis ou transmis s'obtiennent en écrivant la continuité des composantes tangentielles des champs (électriques et magnétiques) à l'interface. Par exemple si nous cherchons à exprimer les facteurs de réflexion $\mathrm{r}_{\mathrm{p}}$ et de transmission $t_{p}$ pour la polarisation linéaire parallèle au plan d'incidence (figure 1), la continuité des composantes tangentielles du champ conduit a :

$$
E_{\text {ip }} \cos i_{1}+E_{r p} \cos i_{1}=E_{t p} \cos i_{2}
$$

si $r_{p}=\frac{E_{r p}}{E_{i p}} t_{p}=\frac{E_{t p}}{E_{i p}}$ on aura :

$$
\left(1+r_{p}\right) \cos i_{1}=t_{p} \cos i_{2}
$$

Pour cette même polarisation, compte tenu de la continuité du champ magnétique qui est perpendiculaire au plan d'incidence, on trouve : $n_{1}\left(1-r_{p}\right)=n_{2}^{t} p(2)$ et en combinant (1) et (2)

$$
\begin{gathered}
r_{p}=\frac{n_{1} / \cos i_{1}-n_{2} / \cos i_{2}}{n_{1} / \cos i_{1}+n_{2} / \cos i_{2}} \\
t_{p}=\frac{n_{1}}{n_{2}}\left(1-r_{p}\right)
\end{gathered}
$$

On montrerait de même que pour la polarisation (s) perpendiculaire au plan d'incidence 


$$
\begin{gathered}
r_{8}=\frac{n_{1} \cos i_{1}-n_{2} \cos i_{2}}{n_{1} \cos i_{1}+n_{2} \cos i_{2}} \\
t_{1}=1+r_{8}
\end{gathered}
$$

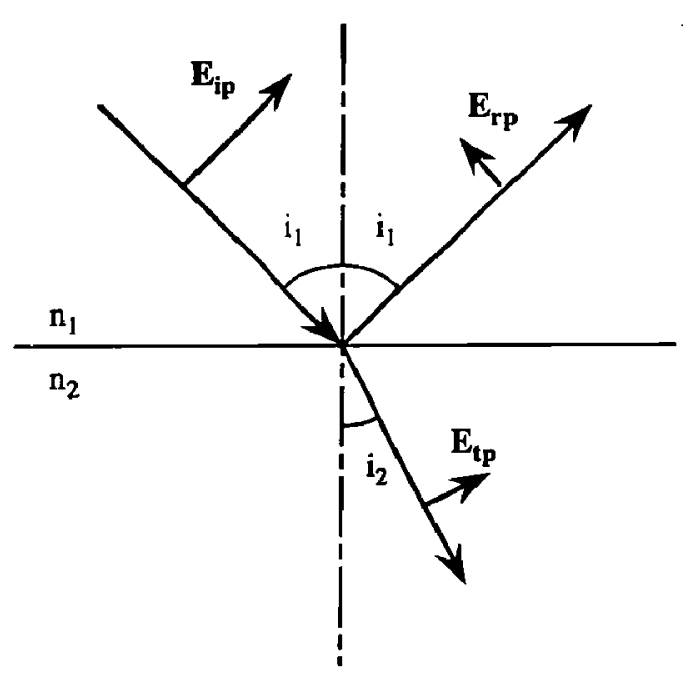

Figure 1 : Calcul des facteurs de réflexion $r_{p}$ et de eransmission $t_{p}$ à l'interface entre deux milieux d'indices $n 1$ et $\mathbf{n}_{2}$.

Ces formules sont très faciles à utiliser, elles permettent de calculer le comportement de systèmes simples (un seul matériau) ou complexes (succession de couches minces) en tenant compte de la loi de la réfraction $\left(n_{1} \sin i_{1}=n_{2} \sin i_{2}\right)$ et des effets d'interférences éventuels.

A titre d'exemple la figure 2 représente les facteurs de réflexion air-eau, air-verre, airgermanium (dans l'infrarouge) en fonction de l'angle d'incidence.

L'utilisation des formules ci-dessus et l'observation des courbes appellent quelques commentaires:

- Les états "propres" de la polarisation (ceux qui sont conservés par réflexion ou transmission) correspondent aux polarisations linéaires parallèle ou perpendiculaire au plan d'incidence. Si on s'intéresse au devenir d'un état de polarisation incident quelconque, il faudra décomposer le champ correspondant sur cette base $(p, s)$ puis le recomposer après interaction avec la surface (cf. ellipsométrie dans les exemples ci-dessous).

- Les formules rendent compte des amplitudes des champs, aussi faut-il prendre le carré du module pour la réflexion $R_{p, s}$ et faire attention aux quantités pertinentes (densité d'énergie ou flux total) pour la réfraction (le plus simple est de calculer $T_{p \cdot s}=1-R_{p \cdot s}$ ).

- Ces formules sont applicables avec des valeurs complexes de l'indice, il faut simplement remarquer que lorsque l'incidence est oblique les ondes réfractées (par exemple à l'interface entre un milieu transparent et un milieu absorbant) ne sont plus homogènes (les plans équiphases ne sont plus des plans équiamplitudes). 
- Au delà de l'angle limite ces formules restent valables pour les champs évanescents (qui sont aussi des ondes non homogènes).

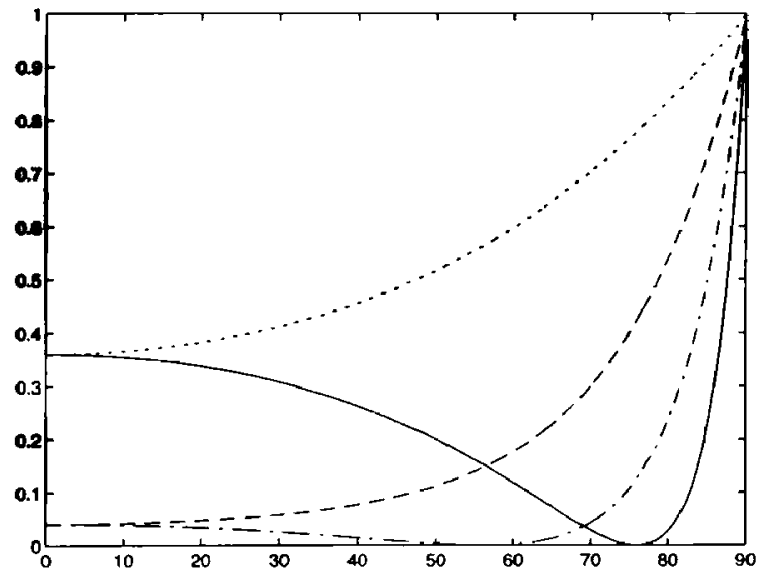

Figure 2 : Facteurs de réflexion (intensité) à l'interface air verre $R_{p}(----)$ et $R_{s}(--)$ dans le visible et air germanium dans l'infrarouge $R_{p}\left(\stackrel{p}{\longrightarrow}\right.$ et $R_{s}(\ldots . .$.$) .$

\subsection{Application : principe de l'ellipsométrie}

Ces quelques notions sur les effets de polarisation aux interfaces vont nous servir à montrer combien la lumière constitue une sonde sensible de ces interfaces et comment de très faibles perturbations très inférieures à la longueur d'onde peuvent être révélées par analyse de l'état de polarisation refléchi ou transmis.

Bien que le schéma proposé ne soit pas le seul possible, la figure 3 nous foumit le principe d'une mesure ellipsométrique : une lumière incidente polarisée à $45^{\circ}$ du plan d'incidence se divise en deux composantes d'égale amplitude $E_{p i}$ et $E_{s i}$ respectivement parallęle et perpendiculaire au plan d'incidence. Chacune de ces composantes sera refléchie par l'échantillon avec les facteurs complexes $r_{p}$ et $r_{S}$. Le rapport des modules tg $\psi$ de ces facteurs complexes fixe la direction du grand axe de l'ellipse représentant le champ réfléchi, la différence des arguments $(\Delta)$ fixe l'ellipticité (rapport des axes).

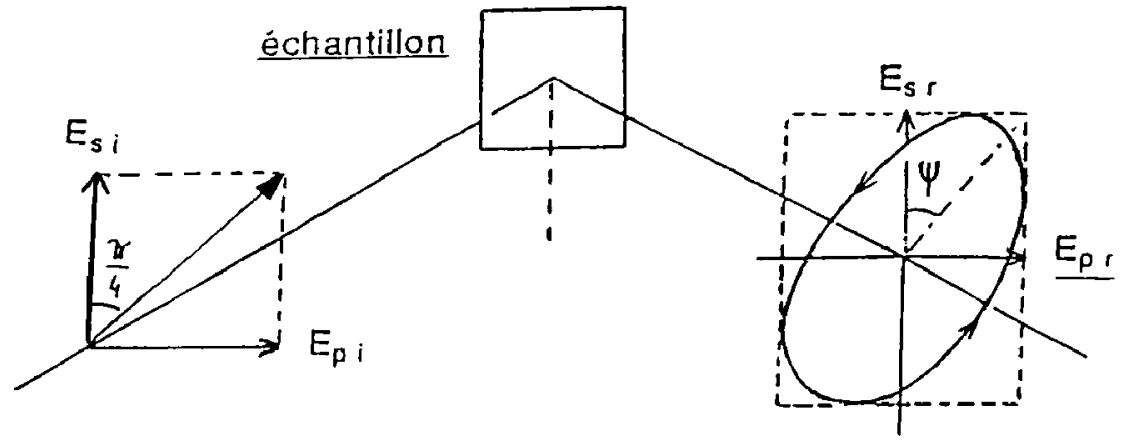

Figure 3 : Principe de l'ellipsométrie. 
L'ellipsométrie est une méthode reconnue d'étude des surfaces et des films minces [4] [5], essayons de voir quelles informations peuvent être déduites de telles mesures :

- Pour un substrat nu, la mesure fournira deux paramètres ( $\psi$ et $\Delta$ ) qui peuvent permettre de trouver les parties réelles et imaginaires de l'indice. Une analyse un peu plus détaillée montre que la précision est sans doute moins bonne que celle que l'on obtient en mesurant la déviation par un prisme sur un diélectrique transparent, mais que la mesure est largement justifiée sur un matériau opaque (ex. semiconducteur ou métal).

- C'est certainement lorsqu'il y a une ou plusieurs couches à la surface d'un matériau que l'ellipsométrie devient incontournable. Cela peut paraître surprenant car chaque mesure ne fournit que deux paramètres alors que nous avons là beaucoup d'inconnues (parties réelles et imaginaires des indices, épaisseurs des couches). Deux approches sont possibles dès que le problème comprend plus d'une couche transparente sur un substrat connu : faire varier I'incidence, et/ou la longueur d'onde.

La variation de l'angle d'incidence est parfois techniquement délicate mais existe de façon automatique sur certains instruments commerciaux. La variation de la longueur d'onde (ellipsométrie spectroscopique) est certainement beaucoup plus répandue aujourd'hui. Couplée à une base de données (sur les indices des matériaux) et à des calculs d'optimisation multiparamètres, c'est une méthode puissante de modélisation des systèmes multicouches.

La figure 4 montre la sensibilité du signal ellipsométrique à un film de silice d'épaisseur subnanométrique sur un substrat de silícium. L'incidence de $76^{\circ}$ correspondrait à l'angle de Brewster si la partie imaginaire de l'indice était nulle.

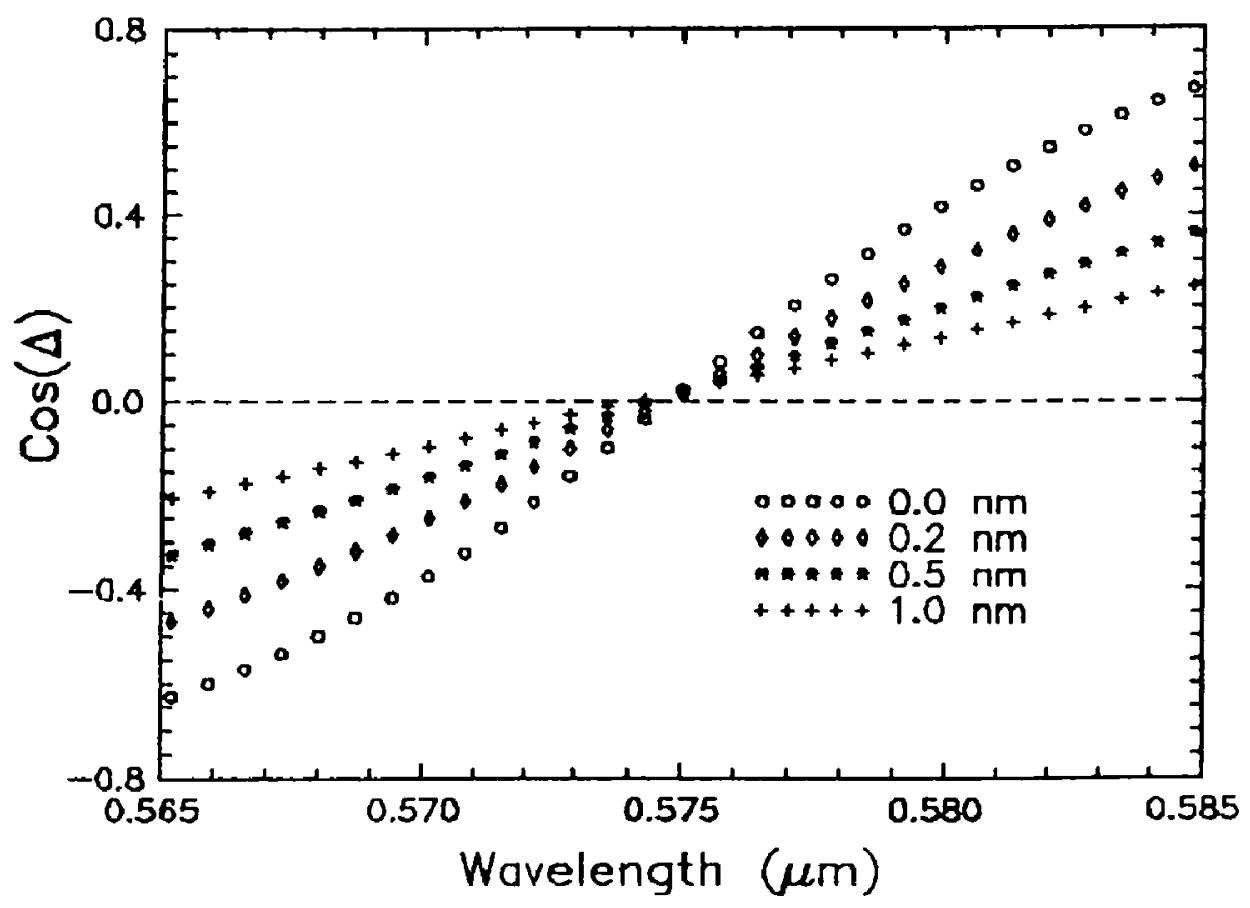

Figure 4 : Variation du signal ellipsométrique ( $\cos \Delta)$ en fonction de l'épaisseur d'un film de silice $\left(\mathrm{S}_{\mathrm{i}} \mathrm{O}_{2}\right.$ amorphe) sur la surface (incidence $76^{\circ}$ ). 
La figure 5 montre le modèle rendant compte de la structure SIMOX (silicium implanté par des ions oxygène qui oxydent $\mathrm{Si}_{\text {en }} \mathrm{SiO}_{2}$ ) en parallèle avec les résultats expérimentaux.
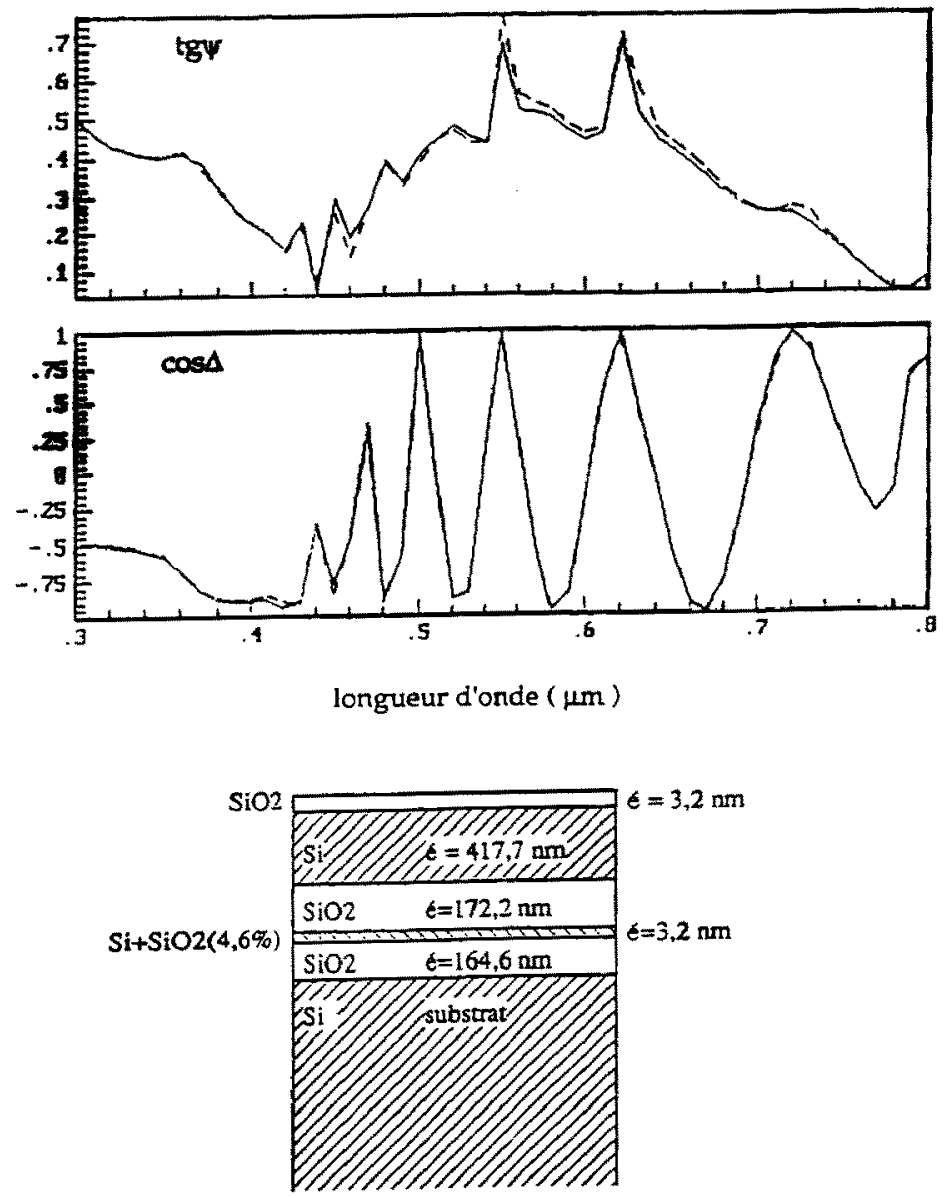

un modele de la structure snMOX

Figure 5 : Résultats expérimentaux d'ellipsométric spectroscopique (-----.) et modèle (- - - ) pour une structure SIMOX

\subsection{Les milieux anisotropes et la polarisation de la lumière}

\subsubsection{Définition.}

Supposons que nous polarisions (électriquement) un milieu diélectrique isotrope. La polarisabilité $\chi$ est un scalaire et $\mathrm{P}_{\mathrm{i}}=\chi \varepsilon_{\mathrm{o}} \mathrm{E}_{\mathrm{i}} \quad \forall \mathrm{i}=\mathrm{x}, \mathrm{y}, \mathrm{z}$. 
Dans un milieu anisotrope on s'attend déjà à ce que les choses soient différentes selon des directions différentes (figure 6), par exemple que, selon l'axe $1, \vec{P}_{1}=\chi_{1} \varepsilon_{0} \vec{E}_{1}$ et que, selon l'axe 2, $\overrightarrow{\mathrm{P}}_{2}=\chi_{2} \varepsilon_{0} \overrightarrow{\mathrm{E}}_{2}$ avec $\chi_{1} \neq \chi_{2}$.

La première conséquence est que si $\vec{E}$ n'est pas parallèle à une des deux directions particulières 1 et $2, \vec{P}$ et $\vec{E}$ ne sont pas colinéaires comme le montre la figure 6 .

En fait, c'est l'absence de colinéarité entre $\vec{P}$ et $\vec{E}$ que nous retiendrons comme définition pour les milieux anisotropes. Par exemple si le champ $\vec{E}$ est appliqué dans la direction $x$, nous aurons 3 composantes pour $\overrightarrow{\mathrm{P}}$ :

$$
\begin{aligned}
& P_{x}=\chi_{x x} \varepsilon_{0} E_{x} \\
& P_{y}=\chi_{y x} \varepsilon_{o} E_{x} \\
& P_{z}=\chi_{z x} \varepsilon_{o} E_{x}
\end{aligned}
$$

et d'une façon plus générale $P_{i}=\sum_{j} \chi_{i j} \varepsilon_{o} E_{j} \quad i, j=x, y, z$. $\chi_{i j}$ est une des 9 composantes du "tenseur des polarisabilités". Ce tenseur est symétrique (6 composantes indépendantes); il peut être ramené à un système d'axes propres (axes principaux $x, y, z)$ pour lequel il est diagonal ( 3 composantes). Ces trois composantes sont différentes $\left(\chi_{x} \neq \chi_{y} \neq \chi_{z}\right)$ pour les milieux de basse symétrie et deux d'entre elles dégénérées lorsque le milieu possède un axe d'ordre supérieur ou égal à 3 (axe optique $\chi_{\mathrm{x}}=\chi_{\mathrm{y}} \neq \chi_{\mathrm{z}}$ ).

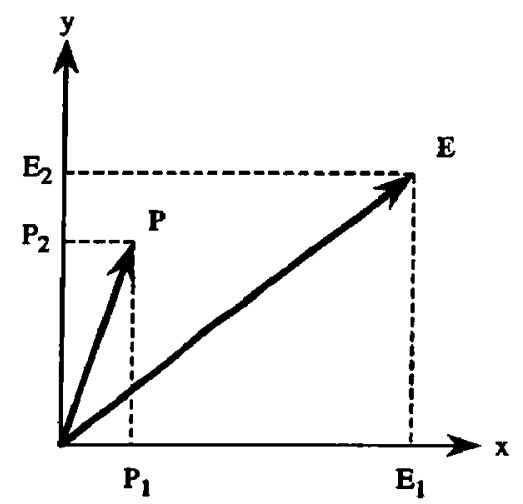

Figure 6 : Polarisation d'un milieu anisotrope.

\subsubsection{Ondes électromagnétiques dans un milieu anisotrope.}

Nous nous limiterons aux milieux électriquement anisotropes, non magnétiques $\left(\mu=\mu_{0}\right)$ pour simplifier la discussion malgré l'importance de ces derniers matériaux pour tout ce qui conceme le stockage et la lecture de l'information par voie optique. 
Nous savons que dans le vide ou dans un diélectrique isotrope le vecteur d'onde $\vec{k}$ et le vecteur de Poynting $\vec{R}$ (rayon), le champ électrique $\vec{E}$ et le déplacement $\vec{D}$, le champ magnétique $\overrightarrow{\mathrm{H}}$ et l'induction $\overrightarrow{\mathrm{B}}$ sont colinéaires 2 à 2 et orthogonaux les uns aux autres (ondes transversales) (figure 7) ; que reste-t-il de cette transversalité des ondes dans les milieux anisotropes?

A partir des équations de Maxwell on montre que si une onde plane peut se propager dans un milieu anisotrope non magnétique, sa structure sera telle que le vecteur de Poynting, le champ électrique et le champ magnétique forment un trièdre trirectangle différent de celui fourni par le vecteur d'onde, le vecteur déplacement et l'induction magnétique (figure 7).

De telles ondes existent-elles?

Si on se fixe une direction du vecteur d'onde $\vec{k}$ (ou du vecteur de Poynting $\vec{R}$ ), on trouve deux directions orthogonales du vecteur déplacement (ou du vecteur champ électrique), chacune étant affectée d'une valeur de l'indice (de la vitesse) solution d'une équation de la forme :

$$
\sum_{i} \frac{k_{i}^{2} n_{i}^{2}}{n^{2}-n_{i}^{2}}=0
$$

où l'indice $\mathrm{i}$ est relatif aux axes $\mathrm{x}, \mathrm{y}, \mathrm{z}$ du tenseur des polarisabilités et $\mathrm{k}_{\mathrm{i}}$ sont les composantes du vecteur d'onde sur ces 3 axes.

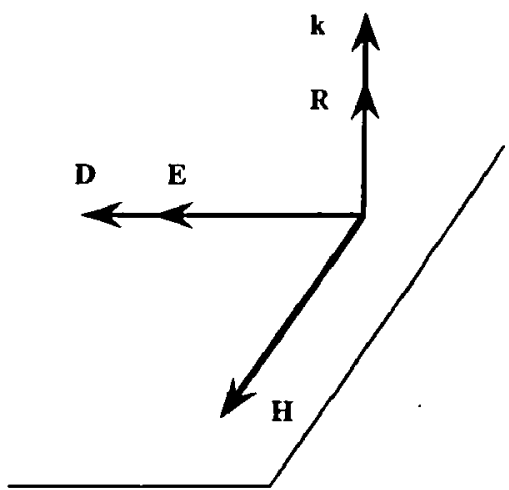

Milieu isotrope

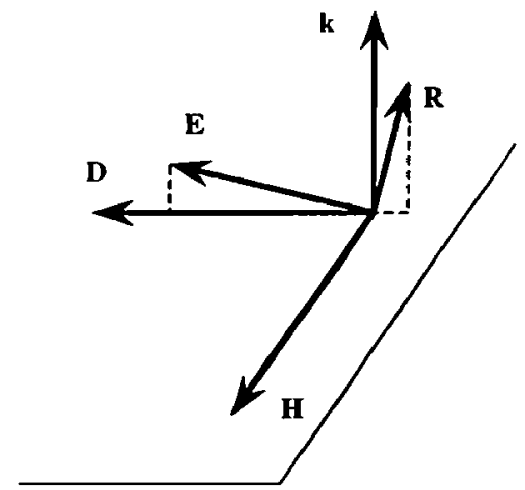

Milieu anisotrope

Figure 7 : Ondes électromagnétiques dans un milieu isotrope et anisotrope.

L'ensemble des valeurs de $n$ (ou v) pour les différentes directions de $\vec{k}$ (ou de $\overrightarrow{\mathbf{R}}$ ) permet de construire une surface à deux nappes : surface des indices (ou des vitesses). 
Cette double valeur de l'indice (ou de la vitesse) pour les états propres de la polarisation à travers les milieux anisotropes est à l'origine de la "double réfraction" que l'on observe facilement lorsque la lumière se propage dans de tels milieux (translation ou déviation et dédoublement des images à travers une lame à faces parallèles ou un prisme) et que les indices ( $n$ ' et $n$ ") correspondant à chacune des polarisations sont suffisamment différents.

Les valeurs de ( $\left.n^{\prime}-n^{\prime \prime}\right)$ les plus typiques sont de l'ordre de $10^{-1}$ (spath), $10^{-2}$ (quartz), $10^{-3}$ (ruban adhésif) à $10^{-4}$ (anisotropie induite sur du verre par une contrainte uniaxiale de $\left.100 \mathrm{~kg} / \mathrm{cm}^{2}\right)$.

Remarque : Il existe des milieux, appelés chiraux, de symétrie particulière (absence de centre d'inversion) pour lesquels les deux "états propres" ne correspondent pas à des polarisations linéaires mais circulaires orthogonales (lumières circulaires droite et gauche). Cette symétrie peut exister à l'échelle microscopique (solution du sucre) ou macroscopique (cristaux de quartz, BGO...). Ces différences d'indice sont beaucoup plus faibles $\Delta n \approx 10^{-6}$.

\subsection{Interférences en lumière polarisée}

On entend par là, interférences entre deux polarisations orthogonales, comme celles qui se propagent dans les milieux anisotropes que nous venons de voir. Comment faire interférer de telles ondes, quelles sont les différences avec les phénomènes d'interférences plus classiques (fentes d'Young, interféromètre de Michelson...), quels montages pratiques peut-on réaliser sur ce principe et quels en sont les avantages? Nous tenterons de répondre à ces questions à travers quelques exemples de montages importants.

\subsubsection{Lames minces biréfringentes.}

Ce sont des lames à faces parallèles taillées, clivées (cristal) ou découpées (polymères) dans des milieux anisotropes. Le terme "mince" signifie simplement ici que les effets de translation des rayons associés à la double réfraction sont négligeables et que seules les différences de marche pour les deux états propres orthogonaux de la polarisation sont à prendre en compte. Les directions correspondantes s'appellent les "lignes neutres" de la lame car la polarisation est conservée selon ces directions.

Comment faire interférer ces deux états de polarisation? Même si la source utilisée ne pose pas de problème de cohérence spatiale ou temporelle (par exemple un laser), nous avons des contraintes qui nous imposent (figure 8):

- de polariser (Polariseur P) la lumière avant la lame, sans cela les phases des deux états de polarisation correspondant aux lignes neutres varieraient de façon aléatoire interdisant tout effet d'interférence.

- de placer un second polariseur (Analyseur A) après la lame biréfringente pour obtenir l'interférence entre la projection des deux vibrations orthogonales sur la direction de ce polariseur.

Soit $\Delta n=n^{\prime \prime}-n^{\prime}=$ biréfringence, $\varphi=\frac{2 \pi \Delta n e}{\lambda}$, e épaisseur de la lame, $\lambda$ longueur d'onde de la lumière. Le rapport entre l'intensité émergente et l'intensité incidente est donné par:

$$
I / I_{o}=\cos ^{2}(\alpha-\beta)-\sin 2 \alpha \sin 2 \beta \sin ^{2} \varphi / 2
$$




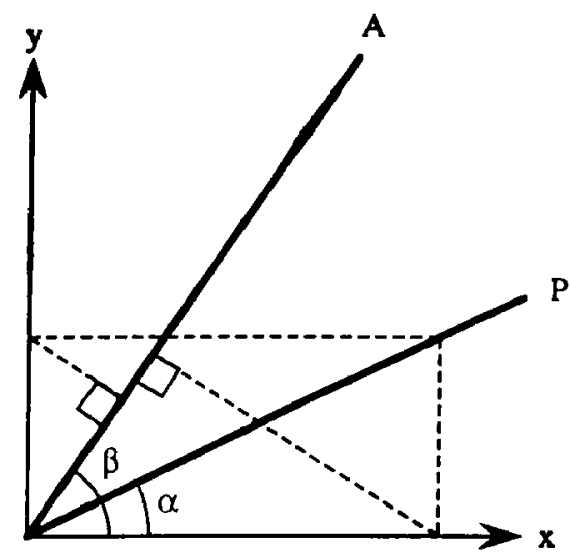

Figure 8 : Interférences en lumière polarisée, $x$ et $y$ sont les directions des lignes neutres.

Dans le cas particulier où les polariseurs sont à $45^{\circ}$ des lignes neutres, ces expressions se simplifient, et on a en particulier :

$$
\begin{gathered}
L / I_{0}=\cos ^{2} \varphi / 2 \text { pour A et } P \text { parallèles } \\
I / I_{0}=\sin ^{2} \varphi / 2 \quad \text { pour A et } P \text { croisés }
\end{gathered}
$$

Nous retrouvons un comportement classique d'interférences à deux ondes d'égale amplitude (contraste maximum $=1$ ) respectivement à centre noir et à centre blanc.

Lorsque de telles lames (par exemple de très fines lames de mica ou du ruban adhésif) sont éclairées en lumière blanche, pour des différences de marche inférieures a la longueur d'onde, on observe des teintes de Newton à centre noir (polariseurs croisés) ou blanc (polariseurs parallèles) qui nous permettent (avec un peu d'entraînement) de quantifier à quelques nm près dans les cas les plus favorables (autour des teintes sensibles) la valeur de la différence de marche.

Rappelons que, comme dans le cas "classique" des interférences à deux ondes (film d'huile sur de l'eau), les couleurs sont dues à la variation du déphasage $\varphi$ avec les longueurs d'ondes $\lambda$ du spectre visible $(\varphi=2 \pi \Delta$ ne $/ \lambda)$.

Pour des différences de marche supérieures à la longueur d'onde on observe des couleurs (succession de rose et de vert) moins quantifiables à l'oeil puis du blanc d'ordre supérieur (correspondant à un spectre cannelé, succession de zones spectrales dont l'intensité varie périodiquement selon les formules 10) qui peut être analysé à l'aide d'un spectromètre.

Pour conclure et insister encore une fois sur la très forte analogie qui existe entre le phénomène d'interférence à deux ondes et ces interférences en lumière polarisée nous pouvons réaliser (en pensée ou matériellement) l'expérience suivante :

Plaçons deux polariseurs linéaires (de qualité interférométrique pour ne pas abîmer le front d'onde) dans les deux bras d'un interféromètre de Michelson (éclairé par un faisceau polarisé monochromatique et collimaté). Si les deux directions de polarisation sont orthogonales (et correspondent au plan d'incidence sur la séparatrice et au plan perpendiculaire pour éviter tout effet d'elliptisation à la réflexion et à la transmission), nous nous trouvons dans une situation analogue à celle qui existe à la sortie d'une lame mince 
biréfringente : nous devons composer deux vibrations orthogonales dont le déphasage dépend de la position relative des miroirs de l'interféromètre.

Nous pouvons ainsi "fabriquer" de la lumière elliptique, circulaire... Comme nous le ferions avec une lame biréfringente d'épaisseur variable.

Pour observer des interférences entre ces deux directions de polarisation il nous faudra placer un autre polariseur (Analyseur) à la sortie du dispositif, après recombinaison des faisceaux issus des deux bras et un polariseur à l'entrée.

Remarque : Cas des milieux chiraux

Ce sont ces milicux pour lesquels les indices $\mathrm{n}_{\mathrm{d}}$ et $\mathrm{n}_{\mathrm{g}}$ correspondant à une lumière circulaire droite et gauche sont différents. Dans ce cas l'expérience d'interférences en lumière polarisée (polariseur-milieu-analyseur) prend une forme particulière puisque la composition des deux vibrations circulaires d'égale amplitude issues de la vibration rectiligne initiale donne après déphasage une vibration rectiligne dont l'axe a tourné d'un angle $\rho=\varphi / 2$ (déphasage $\varphi=2 \pi\left(n_{d}-n_{g}\right)$ e $/ \lambda$. Dans le cas où les milieux sont transparents, on observe simplement un "pouvoir rotatoire" ; lorsque les milieux sont absorbants, le "dichroïsme circulaire" rendra compte de la différence d'absorption correspondant aux deux polarisations circulaires orthogonales.

\section{QUELQUES INSTRUMENTS DE MESURE UTILISANT LA POLARISATION DE LA LUMIERE}

\subsection{Mesure d'un pouvoir rotatoire : quelques approches instrumentales}

Nous disposons des éléments nécessaires pour comprendre le principe et les limites des instruments de mesure des états de polarisation, mesure de biréfringence, de pouvoir rotatoire, ellipsométrie, dichroïsmes circulaire et linéaire...

\subsection{Rappel sur les détecteurs photo-électriques.}

Avant de décrire un instrument rappelons très brièvement quelques notions simples sur les détecteurs photo-électriques qui se trouvent dans une chaîne de mesure entre la source et le système de traitement électronique du signal [5].

2.1.1.1 La sensibilité d'un détecteur est le rapport entre le signal de sortie (en courant ou en tension) et le flux d'entrée (en watt) pour la longueur d'onde utilisée : par exemple une photodiode au silicium fournit un courant de $0,50 \mathrm{~A} / \mathrm{W}$ à $900 \mathrm{~nm}$.

2.1.1.2 Les mesures optiques sont limitées dans leurs performances ultimes par différents effets :

- des effets "technologiques" : dérives électriques, mécaniques, thermiques, des signaux parasites... qui affectent la qualité de la mesure mais peuvent être réduits en prenant un certain nombre de précautions (qualité des composants, compensation des dilatations, blindages optiques ou électromagnétiques, méthode de modulation, moyennage des signaux ....).

- des effets physiques : il s'agit là de causes fondamentales liées aux lois de la physique. Dans le cas de l'optique les "bruits" fondamentaux ont deux origines :

- la nature des sources lumineuses, qui fait que si une source fournit en moyenne $\mathrm{N}$

photons pendant la mesure, la fluctuation est égale à $\sqrt{\mathrm{N}}$. Plus pratiquement si ces photons sont à l'origine d'un courant i de photoélectrons qui génère une tension $\mathrm{V}$ aux bornes d'une résistance $R$, les écarts quadratiques moyens sont donnés par: 


$$
\left\{\begin{array}{l}
\overline{\delta i}^{2}=2 \mathrm{ei} \Delta \mathrm{f} \\
\overline{\delta \mathrm{v}}^{2}=2 \mathrm{eR}^{2} \mathrm{i} \Delta \mathrm{f}
\end{array}\right.
$$

où e est la charge de l'électron et $\Delta \mathrm{f}$ la bande passante (en $\mathrm{Hz}$ ) de l'instrument de mesure. Ce bruit est appelé bruit de photons, bruit Schot ou bruit Schottky. D'importants efforts de recherche sont menés actuellement pour réaliser des sources de photons moins bruyantes, les états du champ électromagnétique correspondants sont appelés états comprimés ("squeezed states") et devraient être utilisés pratiquement dans les domaines de la transmission de l'information (télécommunications) ou de la mesure.

- l'agitation thermique des porteurs de charge dans les éléments dissipatifs (résistances) génère une tension de valeur moyenne nulle mais dont les fluctuations ont pour valeur :

$$
\overline{\delta i}^{2}=\frac{4 k T}{R} \Delta f \quad \overline{\delta v}^{2}=4 k T R \Delta f
$$

Les deux causes de bruit s'additionnent (les valeurs quadratiques s'ajoutent) ; en général à fort flux le bruit de photons domine alors qu'au voisinage d'un flux nul l'agitation thermique fixera le plus petit signal que le détecteur pourra mesurer (signal équivalent au bruit ou "noise equivalent power").

Remarquons enfin que si le courant ou la tension sont amplifiés par un gain G, si i est le courant moyen après amplification le bruit $\left(\sqrt{\overline{\delta^{2}}}\right)$ vaudra $\sqrt{2 \mathrm{eGi} \Delta \mathrm{f}}$.

2.1.1.3 Mesure du pouvoir rotatoire: Méthode quadratique. C'est la méthode la plus simple a priori ; on dispose de deux polariseurs ; lorsqu'ils sont parallèles on mesure un courant $i_{o}$ sur un photomultiplicateur dont le gain est $G$, nous les croisons et mesurons le courant lorsque I'on place la substance active (pouvoir rotatoire $\rho$ ).

En principe on devrait mesurer seulement un courant :

$$
i=i_{o} \sin ^{2} \rho=i_{0} \rho^{2} \text { si l'angle est faible (d'où le nom de détection quadratique) }
$$

mais les imperfections des polariseurs et du montage font qu'un flux parasite qui engendre un courant $\varepsilon^{2} i_{o}$ se superpose au signal précédent.

Le courant du photomultiplicateur est alors :

$$
i=i_{0}\left(\rho^{2}+\varepsilon^{2}\right)
$$

et le bruit $b=\sqrt{\overline{\delta i}^{2}}=\sqrt{2 e G i}{ }_{o}\left(\rho^{2}+\varepsilon^{2}\right) \Delta f$.

Le signal de mesure ${ }_{0} \rho^{2}$ 'est plus accessible lorsqu'il est inférieur au bruit, on prendra pour signal minimum mesurable le signal égal au bruit.

Application numérique : $i_{0}=0,1 \mathrm{~A}, \mathrm{G}=10^{6}, \varepsilon^{2}=10^{-3}, \Delta \mathrm{f}=1 \mathrm{~Hz}$. On trouve $\rho_{\min } \approx 2,410^{-4}$ rad soit moins d'une minute d'angle. 
2.1.1.4 Mesure du pouvoir rotatoire : détection linéaire. Pour éviter l'influence du terme de lumière parasite en $\varepsilon^{2}$ on ajoute au pouvoir rotatoire à mesurer un pouvoir rotatoire magnétique périodique $\theta=\theta_{\mathrm{m}} \sin \omega t$ obtenu par effet Faraday sur une substance transparente (eau, verre...) avec $\rho \ll<\theta_{m}$. Le courant du photomultiplicateur devient : $i=i_{0}\left[(\theta+\rho)^{2}+\varepsilon^{2}\right]$ et on ne mesure que la composante modulée à $\omega$ de ce signal (avec une "détection synchrone" = "lock-in amplifier") qui vaut

$$
S=2 i_{o} \rho \theta_{m}
$$

Le bruit associe au courant moyen $i=i_{0}\left(\varepsilon^{2}+\frac{\theta_{m}^{2}}{2}\right)$ vaudra alors $b=\sqrt{2 e G \Delta f i_{0}\left(\varepsilon^{2}+\theta_{m}^{2} / 2\right)}$.

Application numérique : avec les valeurs choisies ci-dessus et un angle de modulation $\theta_{m}>\varepsilon$ on voit que le bruit est indépendant de $\varepsilon$ et que

$$
\rho_{\min }=\sqrt{\mathrm{eG} \Delta \mathrm{f} / 2 \mathrm{i}_{\mathrm{o}}} \approx 10^{-6} \mathrm{rad}
$$

ce qui représente un gain de plus de deux ordres de grandeur sur le montage précédent.

Par contre si nous n'avions pas été capables de créer une modulation importante telle que $\theta_{\mathrm{m}}^{2}>\varepsilon^{2}$ le gain aurait été plus faible.

D'un point de vue pratique les angles $\theta_{\mathrm{m}}$ sont faibles (quelques degrés) et si le montage ne possède pas une bonne qualité optique (biréfringence de l'échantillon, mauvaise qualité de polariseurs...) il vaut mieux utiliser le montage décrit dans le paragraphe suivant.

2.1.1.5 Mesure d'un pouvoir rotatoire : utilisation d'un modulateur de biréfringence [6]: On interpose entre le polariseur $P$ et l'analyseur $A$ une lame biréfringente variable créant un déphasage périodique $\gamma=\gamma_{\mathrm{m}}$ sin (t)t dont les lignes neutres sont à $45^{\circ}$ de celles du polariseur, la direction de A est également placée à $45^{\circ}$ de celle de P. Soit a l'amplitude du champ après $P$, les amplitudes après le modulateur sont, selon les deux lignes neutres du modulateur, $a / \sqrt{2}$ et $(\mathrm{a} / \sqrt{2}) \mathrm{e}^{\mathrm{i} \gamma}$. Après le pouvoir rotatoire on recueille sur $\mathrm{A}$ l'amplitude

$$
(\mathrm{a} / \sqrt{2})(\cos \rho+\sin \rho \exp (i \varphi))
$$

ou le flux $\frac{\phi_{0}}{2}(1+\sin 2 \rho \cos \varphi)$ auquel on ajoute $\phi_{0} \varepsilon^{2}$. Le courant du photomultiplicateur vaut alors :

$$
\mathrm{i}=\mathrm{i}_{\mathrm{o}}\left[1+2 \varepsilon^{2}+2 \rho \mathrm{J}_{\mathrm{o}}\left(\varphi_{\mathrm{m}}\right)+4 \rho \mathrm{J}_{2}\left(\varphi_{\mathrm{m}}\right) \cos 2 \omega \mathrm{t}+\ldots\right]
$$

$\left(\cos \left(\varphi_{\mathrm{m}} \sin \omega \mathrm{t}\right)=\mathrm{J}_{\mathrm{o}}\left(\varphi_{\mathrm{m}}\right)+2 \mathrm{~J}_{2}\left(\varphi_{\mathrm{m}}\right) \cos 2 \omega \mathrm{t}+2 \mathrm{~J}_{4}\left(\varphi_{\mathrm{m}}\right) \cos 4 \omega \mathrm{t} \ldots\right.$ où les $\mathrm{J}_{\mathrm{m}}$ sont les fonctions de Bessel d'ordre $\mathrm{m}$ ). 
Le signal de mesure $S$ est celui à la pulsation $2 \omega$, signal que l'on maximise par $\mathrm{J}_{2}\left(\mathrm{~J}_{2}=0,5\right.$ pour $\left.\gamma_{\mathrm{m}}=175^{\circ}\right), \mathrm{S}=2 \mathrm{i}_{\mathrm{o}} \rho \mathrm{J}_{2}\left(\rho_{\mathrm{m}}\right)$ et $\mathrm{b}=\sqrt{\mathrm{eG \Delta \textrm {fi } _ { \mathrm { o } }}}$.

Une application numérique utilisant les valeurs fournies ci-dessus conduit a $\rho_{\text {min }}=1,4.10^{-6} \mathrm{rad}$, nous perdons légèrement en sensibilité par rapport au montage prédédent mais les défauts du système $\left(2 \varepsilon^{2}\right)$ jouent beaucoup moins.

Nous voyons à travers ces quelques exemples combien les méthodes de modulation sont utiles pour atteindre les limites physiques de sensibilité des montages sans être gênés par les imperfections des optiques.

Retenons enfin qu'un pouvoir rotatoire de l'ordre du microradian correspond à une différence de marche $\Delta$ ne de l'ordre de $2.10^{-13} \mathrm{~m}$ a la longueur d'onde de $0,6 \mu \mathrm{m}$. Cette sensibilité a été obtenue avec un courant de photocathode de $10^{-7} \mathrm{~A}$ amplifié a $0,1 \mathrm{~A}$ soit environ $1 \mu \mathrm{W}$ de Iumière incidente.

Soulignons enfin combien la mesure du pouvoir rotatoire est importante industriellement pour calculer la concentration de sucre d'un "jus" (betterave ou raisin) et dans les laboratoires de chimie ou de biologie pour analyser des molécules actives.

\subsection{Interférences en lumière polarisée et topographie picométrique}

Nous avons vu avec l'ellipsométrie ou avec la mesure du pouvoir rotatoire que les différences de marche que l'on pouvait atteindre avec des dispositifs photo-électriques se situaient dans le domaine picométrique $\left(1 \mathrm{pm}=10^{-12} \mathrm{~m}\right)$.

II s'agit là de différences de marche statiques et non de variations périodiques ou impulsionnelles qui sont généralement faciles à mesurer avec les moyens de détection habituels (détection synchrone ou oscilloscope moyenneur) car les dérives des signaux "moyens" (par exemple la différence de marche entre les deux bras d'un interférométre de Michelson) influent peu sur les signaux.

D'un point de vue pratique il est très difficile d'assurer la stabilité mécanique ou thermique $d^{*}$ un interféromètre de Michelson pour lequel les chemins optiques dans chacun des bras sont d'une dizaine de $\mathrm{cm}$ à mieux que quelques nanomètres (ceci impose déjà d'éviter des différences de température supérieures au centième de degré) alors que dans les montages en lumière polarisée les deux ondes suivent des chemins identiques et sont donc peu affectées par les dérives mécaniques ou thermiques du montage. Dans les montages du type Nomarski (cf. cours de M. Roblin) le prisme de Wollaston sépare "légèrement" les faisceaux associés aux deux composantes orthogonales de la lumière susceptibles d'interférer, aussi ces montages sont-ils relativement stables et performants. Si on y ajoute un système de modulation et une détection photo-électrique la sensibilité picométrique peut être atteinte sans trop de difficultés.

\subsubsection{Microscopie picométrique : l'approche monodétecteur.}

La figure 9 décrit le principe de ce microscope [7]. On part d'un microscope classique avec son objectif (travaillant à l'infini dans l'espace image), la lentille de tube et l'oculaire. On place au niveau du cercle oculaire un biprisme de Wollaston en quartz qui fournit à partir du faisceau collimaté issu de la diode laser polarisée, à $45^{\circ}$ du plan de la figure, deux faisceaux collimatés faisant un (petit) angle et dont les polarisations sont orthogonales. Ces deux faisceaux se focalisent selon deux taches de diffraction dans le plan de l'objet. Après réflexion les deux faisceaux se recombinent sur le Wollaston, sont réfléchis par un cube séparateur et traversent un modulateur de biréfringence (du type de celui décrit dans la dernière version du polarimètre) dont les axes sont respectivement parallèle et perpendiculaire au plan de la figure et un polariseur dont l'axe est à $45^{\circ}$ de ce plan. 
Ce montage a pour but de mesurer la différence de marche entre deux polarisations orthogonales, il est identique à un montage de mesure de la biréfringence, ici les différences de phase $\varphi$ entre les deux faisceaux orthogonalement polarisés sont dues à la différence de hauteur entre les deux spots sur l'échantillon $\left(\varphi_{\mathrm{e}}\right)$ et au décalage du prisme de Wollaston $(\varphi w)$.

Le flux détecté s'écrit

$$
\begin{gathered}
I(t)=\frac{I_{0}}{2}\left(1+\cos \left(\varphi+\varphi_{m} \sin 2 \pi f t\right)\right) \\
=\frac{I_{0}}{2}\left[1+J_{0}\left(\varphi_{m}\right) \cos \varphi-2 J_{1}\left(\varphi_{m}\right) \sin \varphi \sin 2 \pi f t+2 J_{2}\left(\varphi_{m}\right) \cos \varphi \cos 4 \pi f t+\ldots\right]
\end{gathered}
$$

où $\varphi_{m}$ est l'amplitude du déphasage introduit par le modulateur et $\varphi=\varphi_{\mathrm{e}}+\varphi_{\mathrm{w}}$ somme des déphasages introduits par l'échantillon et le prisme de Wollaston (rappelons qu'un tel biprisme introduit un déphasage lorsqu'il est translaté) [8].

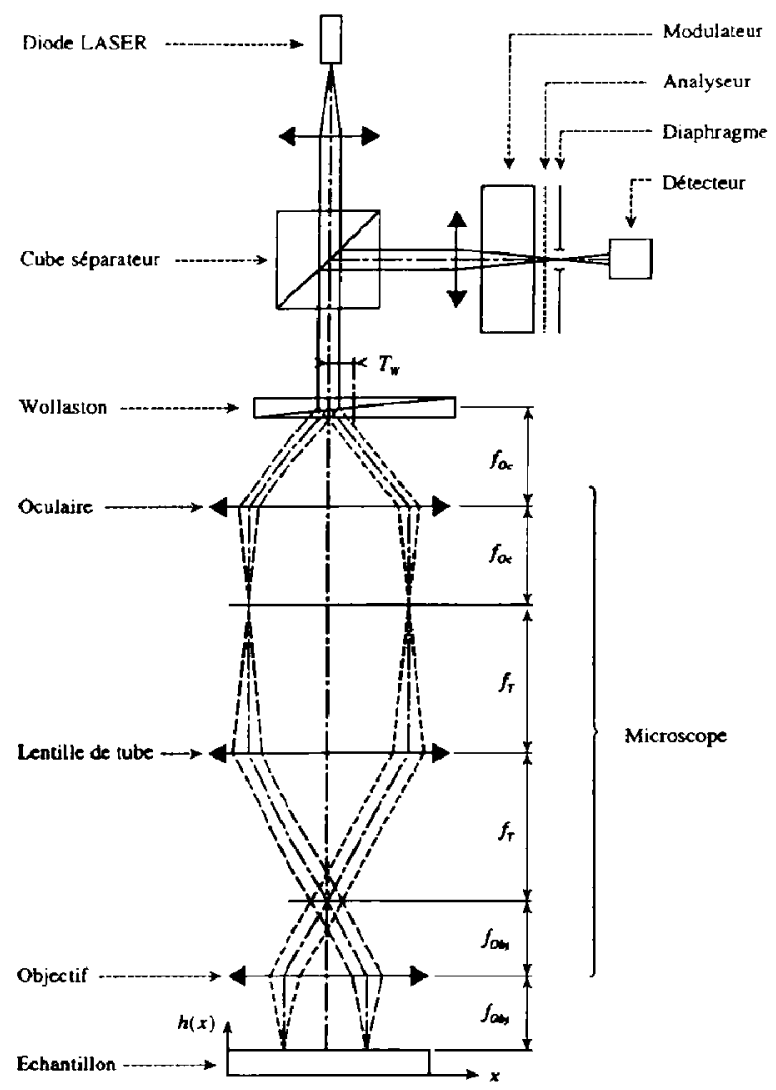

Figure 9 : Principe du profilomètre à polarisation, le signal issu du délecteur est mesuré par une détection synchrone à la fréquence du modulateur 
Le signal à la fréquence $f$ du modulateur, mesuré à l'aide d'une détection synchrone, permet la détermination de $\sin \varphi$ donc du déphasage introduit par l'échantillon.

La figure 10 montre le bruit sur un temps de $500 \mathrm{~s}$ (soit environ 10 fois le temps nécessaire à l'enregistrement d'un profil), ce bruit est inférieur à $1 \mathrm{pm} \mathrm{rms,} \mathrm{ce} \mathrm{qui} \mathrm{montre} \mathrm{la}$ bonne stabilité de ce système différentiel, ce résultat se situe plus d'un ordre de grandeur au dessous du bruit de photons.

Le signal enregistré, en effectuant un balayage sur une ligne à la surface de l'échantillon, étant un signal différentiel il faut le traiter pour remonter au profil réel.

Ce traitement est une déconvolution. On montre que la transformée de Fourier de ce que l'on enregistre est le produit du profil réel tel qu'il apparaît compte tenu de la résolution du microscope, par une fonction sinus.

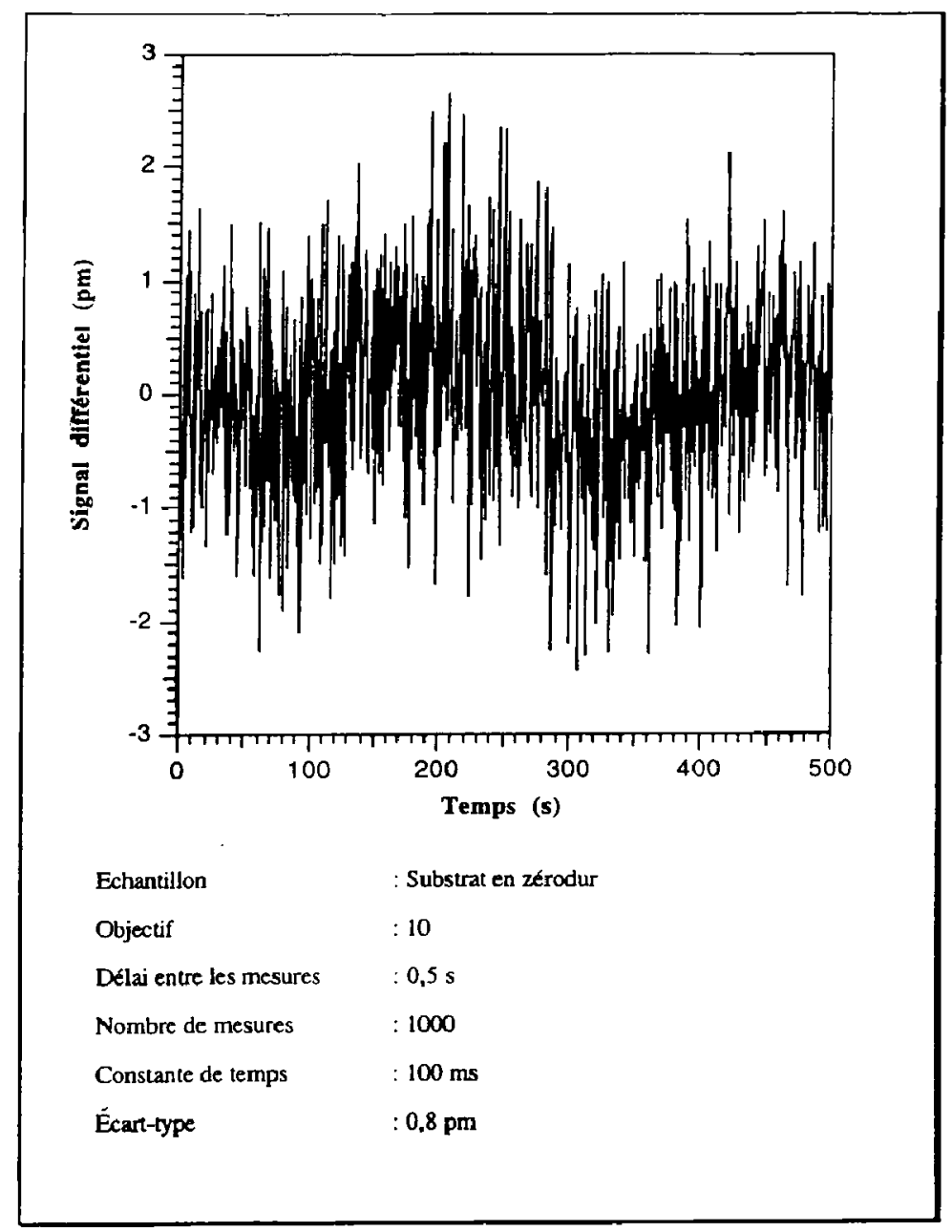

Figure 10 : Mesure de stabilité du signal du microscope interférentiel à polarisation. La valeur RMS du signal est de $0.8 \mathrm{pm}$. 
A titre d'exemple la figure 11 représente le profil d'un substrat de miroir de gysolaser dont on voit que la rugosité est inférieure à $\pm 0,1 \mathrm{~nm}$ sur $1 \mathrm{~mm}$.

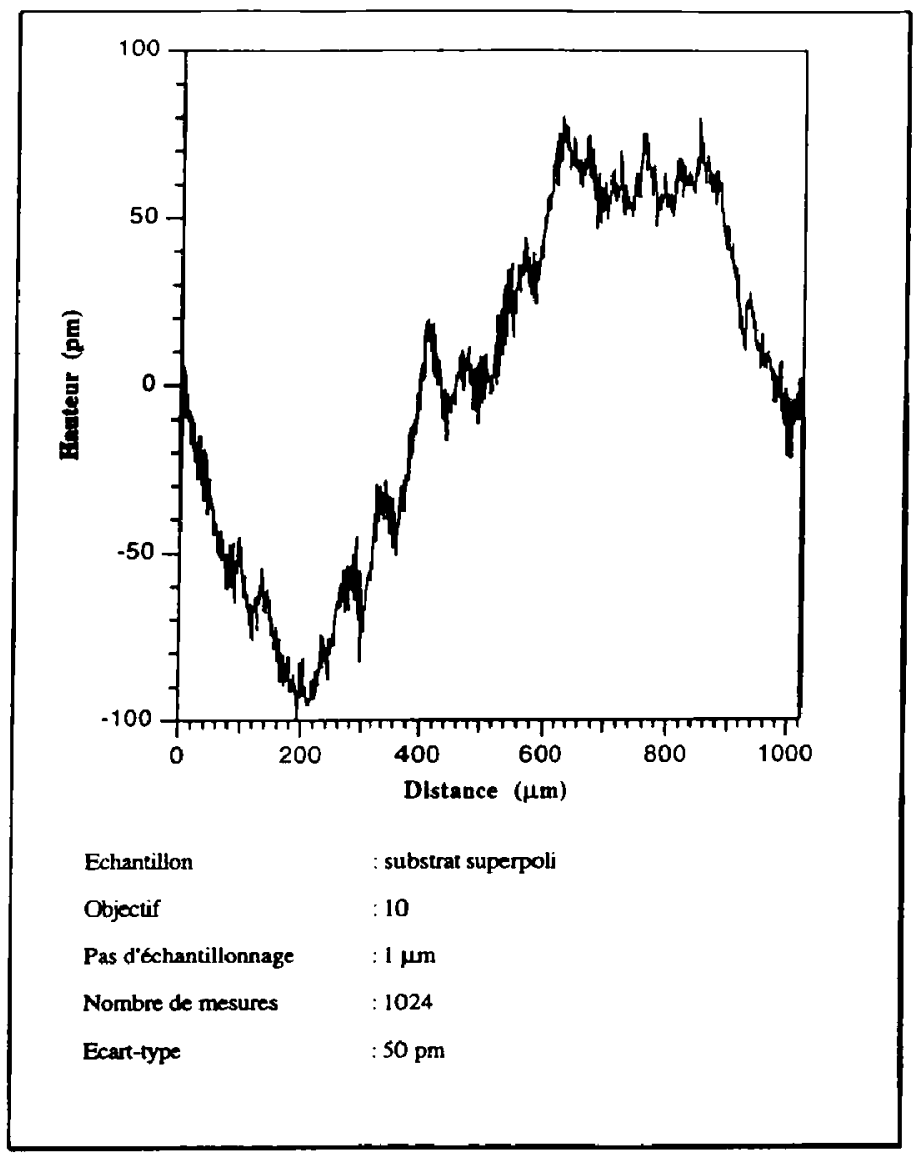

Figure 11 : Profil (déconvolué) d'un substrat superpoli.

Comme nous le rappelions ci-dessus ce montage effectue "presque" une mesure de biréfringence, en effet seul le prisme de Wollaston écarte légèrement les faisceaux et sans ce composant le microscope peut être utilisé pour mesurer la biréfringence locale par réflexion.

\subsubsection{Microscopie picométrique : l'approche multidétecteur.}

Avec le montage précédent acquérir une ligne prenait environ une minute et il était exclu de faire une image de quelques centaines de lignes en conservant les performances du montage : sur un temps aussi long le système ne peut conserver sa stabilité mécanique et thermique sans précautions qui lui feraient perdre sa simplicité.

Aussi avons-nous transposé le principe de la mesure sur un système Nomarski commercial. Ce microscope existe "en standard" au catalogue de tous les fabricants (nous avons travaillé sur un microscope Olympus) et on sait (cf Cours de M. Roblin) qu'avec un tel 
système un observateur averti peut mesurer des différences de marche de l'ordre du nanomètre.

En y ajoutant le modulateur de biréfringence (pour moduler la différence de marche entre les deux polarisations), une caméra $\mathrm{CCD}$ et un nouveau principe de détection synchrone [9] nous avons pu obtenir des performances très voisines de celles du système monodétecteur mais avec une vitesse d'acquisition accrue de deux à trois ordres de grandeur (figure 12).

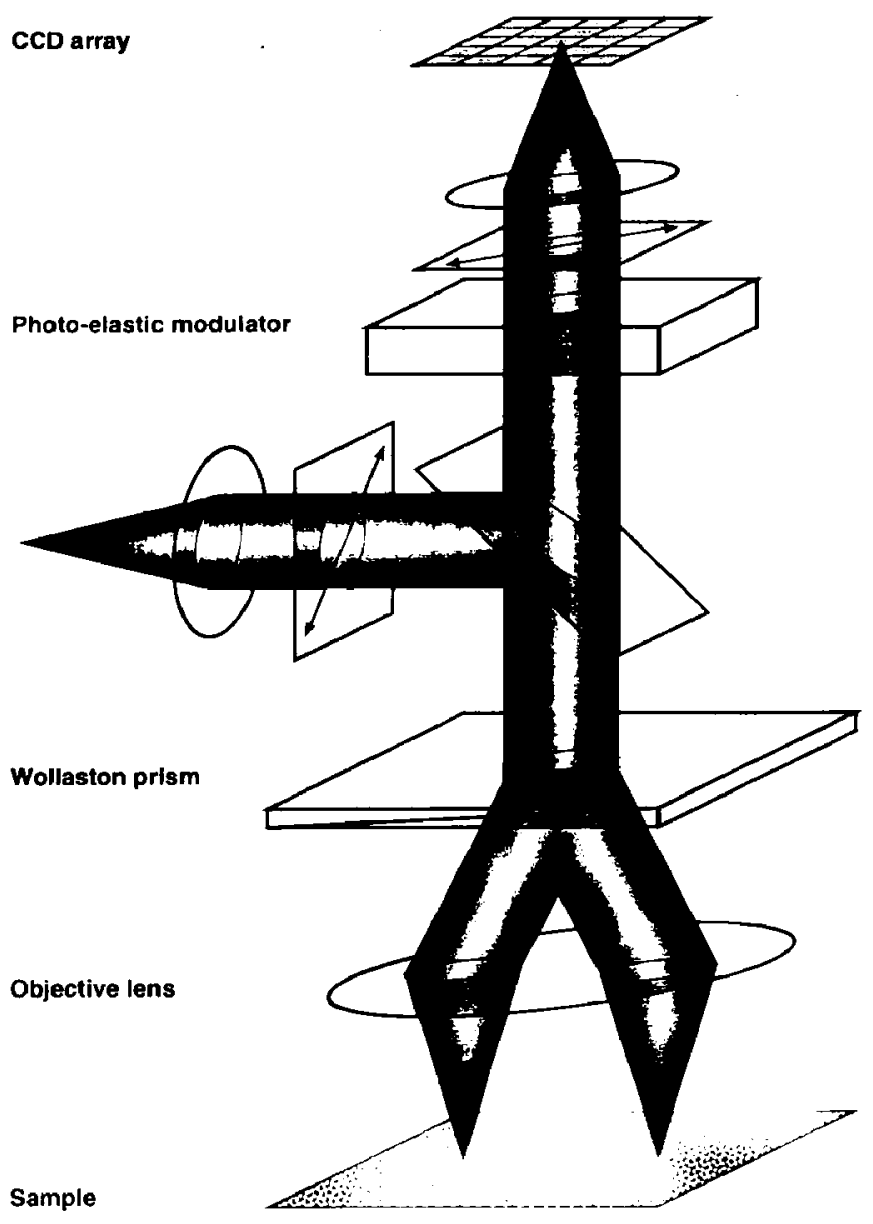

Figure 12 : Microscope Nomarski à modulation de polarisations et détection synchrone multiplex ée.

Pour illustrer, dans le domaine de l'optique, les performances de ce montage et les progrès réalisés dans la fabrication des miroirs multidiélectriques la figure 13 représente la rugosité d'un miroir fabriqué en 1993 et la figure 14 celle d'un miroir fabriqué en 1995.

En 1996 les meilleurs miroirs dont nous disposons ne montrent plus de structures apparentes mais simplement de rares défauts locaux. Ces événements rares sont responsables 
des performances des miroirs au niveau de la diffusion et les valeurs statistiques habituellement utilisées n'ont plus alors de sens.
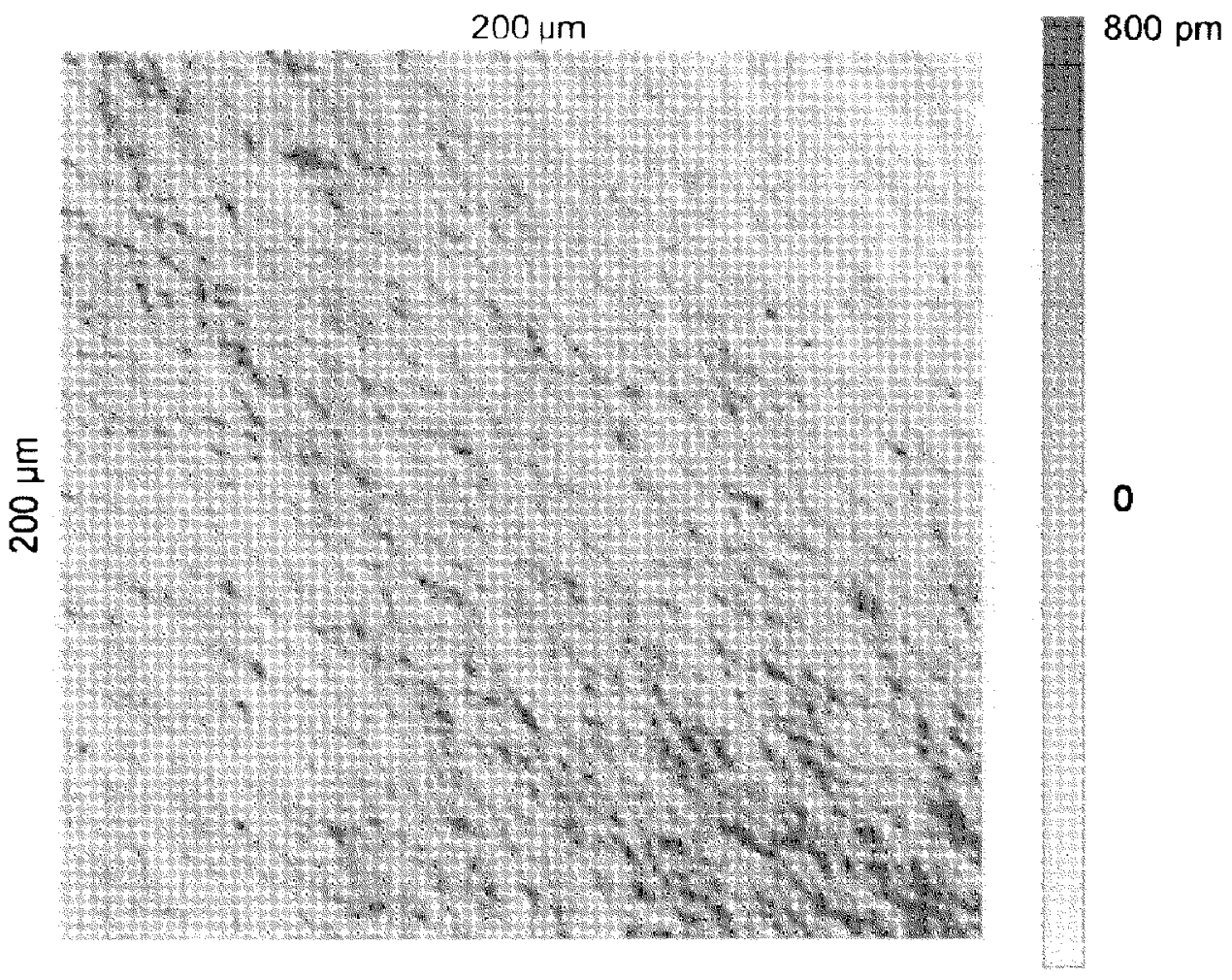

$-800 \mathrm{pm}$

Figure 13 : Rugosité apparente (topographie + texture) d'un miroir réalisé en 1993 (IPNL).

\section{CONCLUSION}

Même si parfois la manipulation des champs polarisés est un peu lourde (nous ne l'avons pas évoquée ici et renvoyons aux ouvrages spécialisés $[10,11,12]$ ), il faut admettre que la mesure de très faibles différences de marche avec des schémas expérimentaux voisins de ceux que l'on utilise dans les polarimètres ou dans les systèmes de mesure de la biréfringence est bien plus simple qu'avec les interféromètres traditionnels.

Les techniques de modulation et de détection synchrone s'avèrent précieuses par les performances qu'elles apportent aux montages, et le fait de pouvoir les utiliser en parallele sur des images entières les rendent très prometteuses pour de futures applications métrologiques. 

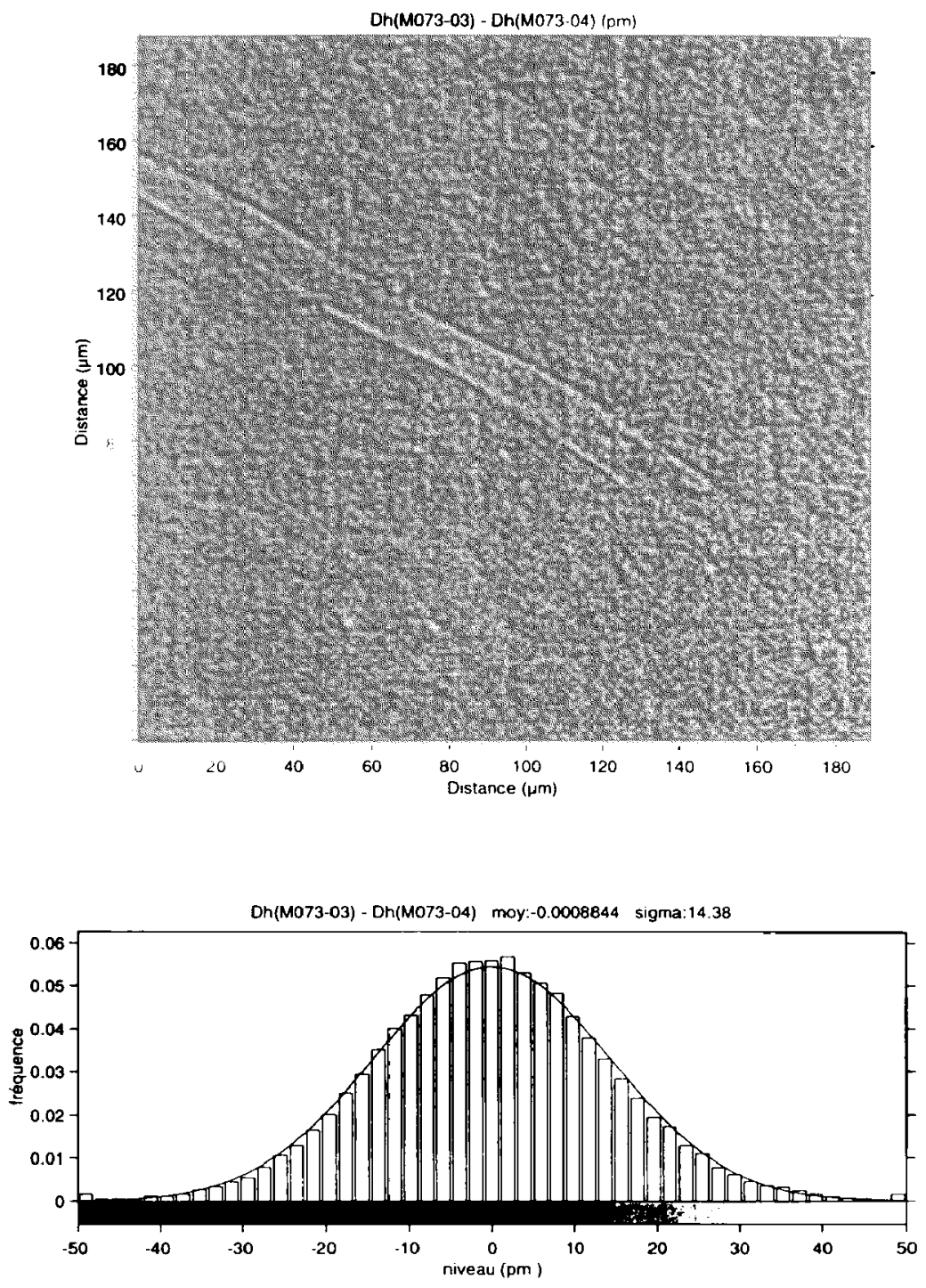

Figure 14 : Rugosité apparente et défaut local d'un miroir réalisé en 1995 (IPNL). La valeur rms de la rugosité est inférieure à $15 \mathrm{pm}$, le bruit de l'instrument est de 7 a $8 \mathrm{pm}$ rms.

\section{Références}

[1] Cohen-Tannoudji C., Dupont-Roc J., Grynberg G., Processus d'interaction entre photons et atomes,(Editions du CNRS, Paris, 1988)

[2] Bruhat G., Optique (Masson, réédité en 1992)

[3] Huard S., Polarisation de la lumière (Masson 1994) 
[4] Bernoux F., Piel J.P., Lecat J.H. et Stehlé J.L., Ellipsométrie, Techniques de l'Ingénieur, traité Mesures et Contrôle

[5] Desvignes F., Détection et détecteurs de Rayonnement Optiques (Masson 1987)

[6] Badoz J., Billardon M., Canit J.C., Russel M.F., Sensitive devices to determine the state and degre of polarization of a light beam using a birefringent modulator, J. of Optics, 8 (1977), p. 373-384

[7] Gleyzes P., Boccara A.C., Profilomérrie picométrique par interférence de polarisation I L'approche monodétecteur, J. of Optics, 25 (1994), p. 207-224

[8] Françon M, et Mallick S., Polarization interferometers (John Wiley, 1971)

[9] Gleyzes P., Guernet F., Boccara A.C., Profilométrie picométrique II - L'approche multidétecteur et la détection synchrone multiplexée, $J$. of Optics, 26 (1995), p. 251-265

[10] Shurcliff W.A., Polarized light (Harvard University Press, Cambridge, 1962)

[11] Collet E., Polarized light (Marcel Dekker, 1993)

[12] Boccara A.C., Pickering C., Rivory J., Spectroscopic Ellipsometry (Elsevier, 1993) 